\title{
Trp RNA-Binding Attenuation Protein: Modifying Symmetry and Stability of a Circular Oligomer
}

\author{
Oliver W. Bayfield ${ }^{1}$, Chao-Sheng Chen ${ }^{10}$, Andrea R. Patterson ${ }^{2}$, Weisha Luan ${ }^{1}$, Callum Smits ${ }^{1}$, \\ Paul Gollnick ${ }^{2}$, Alfred A. Antson ${ }^{1 *}$
}

1 York Structural Biology Laboratory, Department of Chemistry, University of York, York, United Kingdom, 2 Department of Biological Sciences, State University of New York, Buffalo, New York, United States of America

\begin{abstract}
Background: Subunit number is amongst the most important structural parameters that determine size, symmetry and geometry of a circular protein oligomer. The L-tryptophan biosynthesis regulator, TRAP, present in several Bacilli, is a good model system for investigating determinants of the oligomeric state. A short segment of C-terminal residues defines whether TRAP forms an 11-mer or 12-mer assembly. To understand which oligomeric state is more stable, we examine the stability of several wild type and mutant TRAP proteins.

Methodology/Principal Findings: Among the wild type B. stearothermophilus, B. halodurans and B. subtilis TRAP, we find that the former is the most stable whilst the latter is the least. Thermal stability of all TRAP is shown to increase with Ltryptophan concentration. We also find that mutant TRAP molecules that are truncated at the C-terminus - and hence induced to form 12-mers, distinct from their 11-mer wild type counterparts - have increased melting temperatures. We show that the same effect can be achieved by a point mutation S72N at a subunit interface, which leads to exclusion of $C$-terminal residues from the interface. Our findings are supported by dye-based scanning fluorimetry, CD spectroscopy, and by crystal structure and mass spectrometry analysis of the B. subtilis S72N TRAP.

Conclusions/Significance: We conclude that the oligomeric state of a circular protein can be changed by introducing a point mutation at a subunit interface. Exclusion (or deletion) of the C-terminus from the subunit interface has a major impact on properties of TRAP oligomers, making them more stable, and we argue that the cause of these changes is the altered oligomeric state. The more stable TRAP oligomers could be used in potential applications of TRAP in bionanotechnology.
\end{abstract}

Citation: Bayfield OW, Chen C-S, Patterson AR, Luan W, Smits C, et al. (2012) Trp RNA-Binding Attenuation Protein: Modifying Symmetry and Stability of a Circular Oligomer. PLoS ONE 7(9): e44309. doi:10.1371/journal.pone.0044309

Editor: Sergey Korolev, Saint Louis University, United States of America

Received May 14, 2012; Accepted August 1, 2012; Published September 6, 2012

Copyright: (c) 2012 Bayfield et al. This is an open-access article distributed under the terms of the Creative Commons Attribution License, which permits unrestricted use, distribution, and reproduction in any medium, provided the original author and source are credited.

Funding: This work was supported by the Engineering and Physical Sciences Research Council studentship 2011 and the Department of Chemistry Vacation Bursary Scheme 2011 to OWB, Wild Fund awards to C-SC and WL, by National Science Foundation Molecular and Cellular Biology grant 1019960 to PG and by the Wellcome Trust fellowship 081916 to AAA. The funders had no role in study design, data collection and analysis, decision to publish, or preparation of the manuscript.

Competing Interests: The authors have declared that no competing interests exist.

*E-mail: fred.antson@york.ac.uk

a Current address: Division of Immune Cell Biology, National Institute for Medical Research, Mill Hill, London, United Kingdom

\section{Introduction}

Cyclic protein oligomers pose promise as the basis for engineering molecular machines, including those that operate by rotation of one or more components about a principal axis. Their suitability, owing to their unique geometry, is evident by their frequent occurrence in key bio-mechanical and regulatory roles. Examples include circular protein oligomers found in flagellar motors [1], the ATP synthase [2] and the molecular motor of tailed bacteriophages which translocate DNA into the capsid during viral particle assembly [3-5]. The stoichiometry of the MSring and C-ring in flagellar motors are variable [6]. Likewise, the central component of the bacteriophage DNA-translocating motor can also exist in different oligomeric states [5]. This demonstrates a general problem associated with instability of circular oligomers and variation in their oligomeric states when they are extracted from their natural environment. Engineering stable molecular devices of suitable size, geometry, and hence subunit number is of interest for applications in molecular biology and medicine. For example, applications of viral DNA-translocating devices in the transfer of genetic information across biological membranes are attractive. Recent studies aimed at the incorporation of a portal protein into lipid bilayers look particularly promising [7].

The tryptophan RNA-binding attenuation protein, TRAP, possesses similar geometric features to other circular assemblies, being assembled of multiple subunits and containing a central tunnel. Typically composed of 11 or 12 identical subunits, with a molecular weight of 90.6 to $101.9 \mathrm{kDa}[8,9]$, TRAP regulates Ltryptophan biosynthesis genes by attenuation in many Bacilli [10]. When activated by increased levels of L-tryptophan - bound to receptor sites nestled between adjacent subunits - TRAP binds single stranded RNA at the leader region of the $\operatorname{trpEDCFBA}$ operon transcript. This modulates terminator hairpin formation 
and so downregulates L-tryptophan biosynthesis [8]. In this study TRAP is employed as a model for investigating the influences of oligomeric state and stability of such assemblies. To date TRAP has already alluded to its value in bionanotechnology, showing that mutations to residues protruding into the central cavity allow gold nanoparticle binding [11]. Nanotubes consisting of stacked TRAP rings linked by disulfide bonds have also been shown to selfassemble, in response to the introduction of cysteine residues on the ring-faces orthogonal to the principal axis [12].

TRAP oligomers have previously been reported to be highly thermostable [13]. Predictably, we find that the melting temperature is highest in the case of TRAP from B. stearothermohphilus, a hyperthermophile. The presence of L-tryptophan - and hence the bound holo-TRAP state - has been associated with a greater structural rigidity and integrity [14]. Prior to the recent determination of the $B$. stearothermophilus apo-TRAP crystal structure [15] TRAP was crystallized in the presence of Ltryptophan $[8,9]$. We find that L-tryptophan binds with a $\mathrm{K}_{\mathrm{d}}$ in the $2.8 \mu \mathrm{M}$ to $6.9 \mu \mathrm{M}$ range, with small variation between TRAP from different species. We explore how the presence of Ltryptophan and its' binding affects thermal stability in the context of the three wild type and three mutant TRAP molecules. We also explore the influence of the oligomeric state on thermal stability by comparing wild type 11-subunit and mutant 12-subunit molecules from the same species. Finally, we show how the 11-subunit to 12subunit switch could be induced by the point mutation S72N. The analysis includes crystal structure and native mass spectrometry data on $B$. subtilis S72N TRAP. We find that the increase in subunit number increases the stability of the assembly.

\section{Results}

\section{Crystal Structure and Native Mass Spectrometry of $B$. subtilis S72N TRAP}

We previously demonstrated the importance of the G-terminus in oligomer formation during B. subtilis TRAP assembly [9]. However, this work required deletion of the C-terminus to increase the subunit stoichiometry and we questioned if a more subtle mutation at the C-terminus could induce formation of a 12subunit assembly. Based on the previous structural work, we chose the S72N mutation because we expected that the bulky side chain at this position would result in the exclusion of N72 and following C-terminal residues from the subunit interface. The crystal structure of $B$. subtilis S72N TRAP, determined at $2.7 \AA$, reveals a circular 12-mer assembly (Figure 1), which is similar to previously reported crystal structures of TRAP. In the crystal the 12 subunit assembly is generated by combination of six TRAP subunits, present in the asymmetric unit, with the crystallographic 2-fold axis of the $\mathrm{P} 2{ }_{1} 2_{1} 2$ space group (Table 1). The final electron density maps allowed positioning of all residues, with the exception of the four $\mathrm{N}$-terminal residues and the five $\mathrm{C}$-terminal residues of most subunits, for which no clear electron density has been observed, indicating their flexibility. The structure of the S72N TRAP was compared against the wild type TRAP (Figure 2A), which highlighted change in the conformation of the C-terminus in the mutant protein, starting from position 69 . The weighted $F_{o}-$ $F_{c}$ omit electron density maps for residues E69 and M70 (Figure 2B) demonstrate the absence of interpretable electron density beyond position 70 in all chains, indicating that five Gterminal residues are disordered. The crystal structure compares well with our predictions on the effect of the point mutation, in inducing the removal of the $\mathrm{C}$-terminus from the subunit interface through steric hindrance, thus facilitating rigid-body rotations of subunits for the accommodation of one additional subunit into the ring.

To understand if the 12-mer state of the S72N TRAP observed in the X-ray structure, predominates in solution, we analyzed this protein by native mass spectrometry (Text S1). The 12-mer state was identified as the dominant species in the mass spectra (Figure S1), as opposed to the wild type protein which forms 11-mers [16].

\section{2-mer TRAP Assemblies are More Stable}

Melting temperatures for six TRAP molecules, including three wild type and three mutant TRAP listed in Table 2, were determined at varied L-tryptophan concentrations $(0-100 \mu \mathrm{M})$ using dye-based scanning fluorimetry as described in the methods. Fluorescence versus temperature melting curve data (Figure S2, S3) was averaged between four observations and the midpoints of fitted sigmoidal curves were taken as the melting temperatures, $\mathrm{T}_{\mathrm{m}}$. Melting temperatures (Table S1) were then plotted against Ltryptophan concentration (Figure 3).

As the fluorescent dye could potentially bind in the tryptophanbinding pocket, we employed CD spectroscopy (Text S2) to verify that melting temperatures were not perturbed by interaction with the dye, Figure S2. We note that there is coherence between melting temperatures derived from the two respective techniques.

Increasing the L-tryptophan concentration increases the thermal stability of all the TRAP proteins except for wild type B. subtilis TRAP. B. halodurans TRAP was included as a reference, as it is naturally a 12-mer, without engineering. Throughout the Ltryptophan concentrations for which the $\mathrm{T}_{\mathrm{m}}$ was measured, the wild type $B$. subilis TRAP was least stable, while the $B$. stearothermophilus was most stable. Significantly, the mutant 12 subunit assemblies were at least as stable, but usually more stable than the 11 subunit wild-type, at all L-tryptophan concentrations measured. This stabilization was particularly marked at high Ltryptophan concentrations as the mutant 12-mer subunit TRAPs have a larger increase in $\mathrm{T}_{\mathrm{m}}$ with increasing L-tryptophan concentration than their wild type 11-mer counterparts. For example, from 0 to $100 \mu \mathrm{M}$ L-tryptophan, B. subtilis wild type increases in stability by $0.8^{\circ} \mathrm{C}$, while the $\mathrm{T}_{\mathrm{m}}$ of the $\mathrm{K} 71$ stop and S72N 12-mer mutants increases by $23.2^{\circ} \mathrm{C}$ and $13.3^{\circ} \mathrm{C}$ respectively. The $B$. stearothermophilus wild type TRAP increases in stability by $7.1^{\circ} \mathrm{C}$, whereas the $\mathrm{T}_{\mathrm{m}}$ of E7 1stop TRAP increases by $20.2^{\circ} \mathrm{C}$. The thermal stability of $B$. subtilis wild type TRAP appeared to show little dependence on L-tryptophan concentration up to $100 \mu \mathrm{M}$. We observed that the stability of the wild type 12-mer $B$. halodurans TRAP lies midway between that of mutant 12-mers, B. subtilis K71stop and B. stearothermophilus E7 1stop TRAP (Figure 3).

\section{L-Tryptophan Binding}

The increased stabilization by L-tryptophan of the 12-subunit assemblies, as compared to 11-mers, was unexpected. To investigate this further we measured the affinity of each TRAP for L-tryptophan (Table 2). A comparison of the dissociation constants of the mutant 12-mers to their 11-mer wild type counterparts showed no obvious correlation. Although an 11-mer to 12-mer transition in $B$. subtilis TRAP increases the affinity of binding, this is not observed in B. stearothermophilus TRAP. This suggests stabilization by L-tryptophan is not directly related to affinity towards L-tryptophan.

\section{Discussion}

The conformation of the C-terminus has previously been shown to play a major role in determining whether the 11-mer or the 12- 


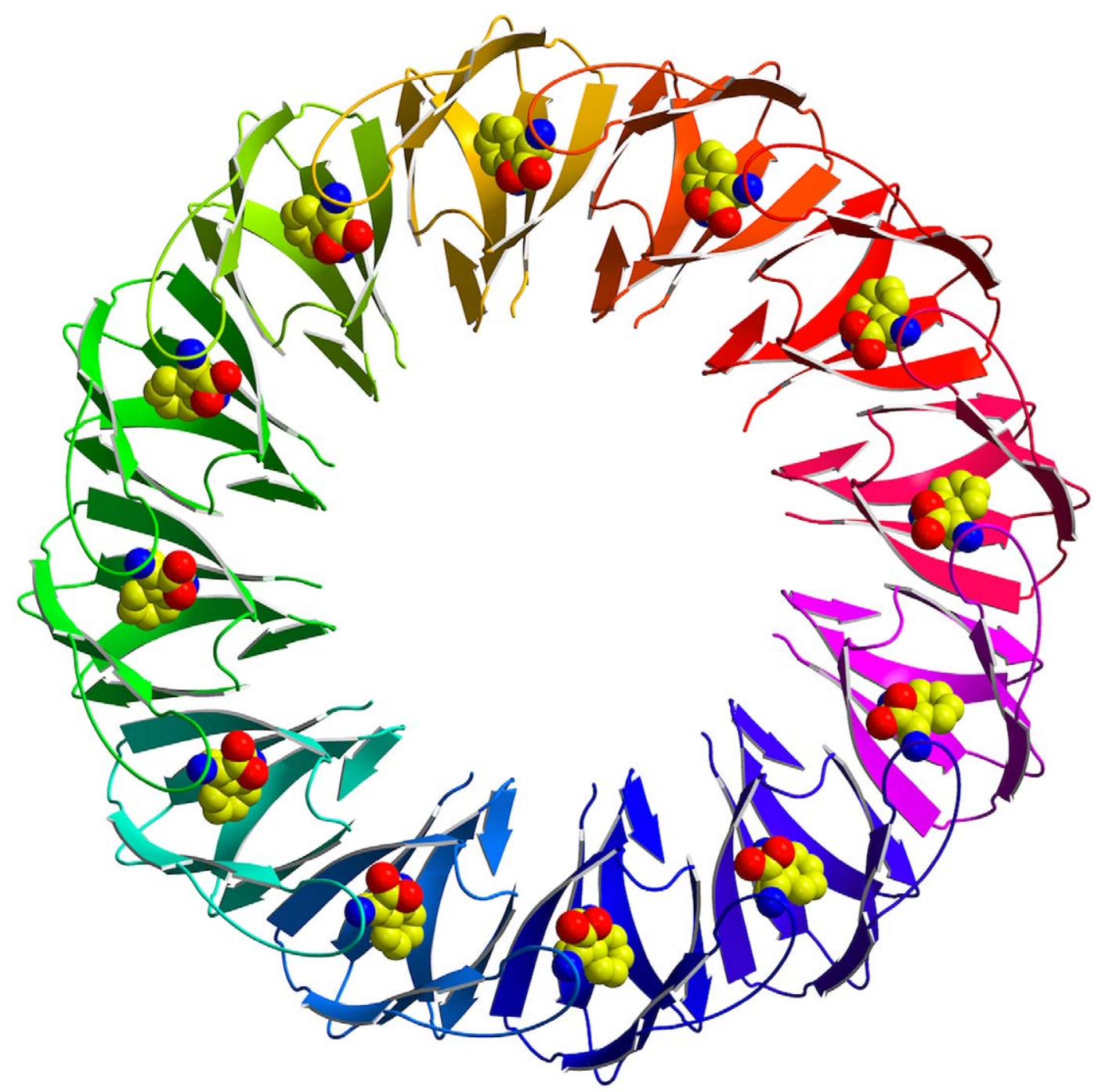

Figure 1. Ribbon diagram of the $B$. subtilis S72N TRAP viewed along the 12-fold axis. Each subunit is shown in a different colour. Ltryptophan molecules are shown as van der Waals models with oxygen atoms in red, nitrogen atoms in blue and carbon atoms in yellow. doi:10.1371/journal.pone.0044309.g001

mer oligomeric state assembles [9], and the new structure of the S72N mutant reinforces this. The relationship between subunit number and thermal stability has for the first time been probed, revealing the 12-mer configuration is more stable than the 11 -mer counterpart.

\section{Comparison of Thermal Stability in Wild Type TRAP}

We compared the thermal stability of $B$. stearothermophilus, $B$. halodurans, and B. subtilis wild type TRAP. The relative thermal stabilities of the oligomers reflect the temperature environments of the respective species' habitats, as might be expected. For example, at $50 \mu \mathrm{M}$ L-tryptophan, the melting temperatures for the respective wild type TRAP are: $B$. stearothermophilus, $81.4^{\circ} \mathrm{C}$; B. halodurans, $75.6^{\circ} \mathrm{C} ;$ B. subtilis, $44.3^{\circ} \mathrm{C}$. These melting temperatures reflect the respective species' hyperthermophilic, thermophilic and mesophilic origins respectively. This relationship appears to hold across all of the L-tryptophan concentration range (Figure 3).

Within these wild type TRAP oligomers, B. stearothermophilus and $B$. subtilis both have 11-mer TRAP, and B. halodurans has 12-mer TRAP. It is apparent therefore that the temperature environment in which the species have evolved is the overriding factor in determining the thermal stability of the wild type TRAP, as we have an 11-mer as both the most and least stable. However, we find that one effect of inducing a change of oligomeric state from 11-mer to 12 -mer by mutations is increased thermal stability, implying that species-specific structural characteristics unrelated to subunit number are not the only determinant of thermal stability.

\section{C-terminal Truncation Induces 11- to 12-mer Transition} and Increases Stability

Comparing the structure of the 12-mer B. halodurans TRAP with that of the 11-mer structures of $B$. subtilis TRAP and $B$. stearothermophilus TRAP, the importance of the C-terminus (residues 71 to 75 ) becomes apparent. In $B$. halodurans TRAP, the nature of the last four amino acids dictate that the G-terminal segment is excluded from the subunit-subunit interface, thereby generating favorable interactions at the outer surface of the oligomer. This is considered as the crucial factor in permitting the higher oligomeric state, which is related to the 11 -mer state by a $2.7^{\circ}$ rigid-body rotation of adjacent subunits [9]. Hence what can be considered 
Table 1. Data collection and refinement statistics for $B$. subtilis S72N TRAP.

\begin{tabular}{|c|c|}
\hline \multicolumn{2}{|l|}{ Data collection } \\
\hline Space group & $\mathrm{P} 2{ }_{1}{ }_{1} 2$ \\
\hline Unit cell & $a=109.4 \AA, b=110.2 \AA, c=46.2 \AA$ \\
\hline Resolution & $25-2.7 \AA(2.80-2.70 \AA)$ \\
\hline No. of reflections & $14253(1025)$ \\
\hline Redundancy & $6.3(4.2)$ \\
\hline$R_{\text {merge }}{ }^{\mathrm{a}}$ \% $\%$ & $9.7(50.2)$ \\
\hline Completeness, \% & $91.9(68.2)$ \\
\hline $1 / \sigma$ & $15.3(2.8)$ \\
\hline Wilson B factor & 86.1 \\
\hline \multicolumn{2}{|l|}{ Refinement } \\
\hline Resolution range & $25-2.72 \AA(2.79-2.72 \AA)$ \\
\hline No. of reflections used in refinement & 13396 \\
\hline $\begin{array}{l}\text { No. of reflections excluded from } \\
\text { refinement }\end{array}$ & 825 \\
\hline$R$ factor $^{\mathrm{b}}, \%$ & $20.9(34.1)$ \\
\hline Free $R$ factor ${ }^{\mathrm{b}}, \%$ & $24.5(38.2)$ \\
\hline \multicolumn{2}{|l|}{ Number of atoms } \\
\hline Protein & 2952 \\
\hline Ligand & 90 \\
\hline Water molecules & 27 \\
\hline Average B factor (protein) & 83.8 \\
\hline Average B factor (solvent) & 82.5 \\
\hline Rmsd bond lengths & $0.009 \AA$ \\
\hline Rmsd bond angles & $1.2^{\circ}$ \\
\hline \multicolumn{2}{|l|}{ Ramachandran plot } \\
\hline Most favored regions, \% & 97.8 \\
\hline Additionally allowed regions, \% & 2.2 \\
\hline \multicolumn{2}{|c|}{ 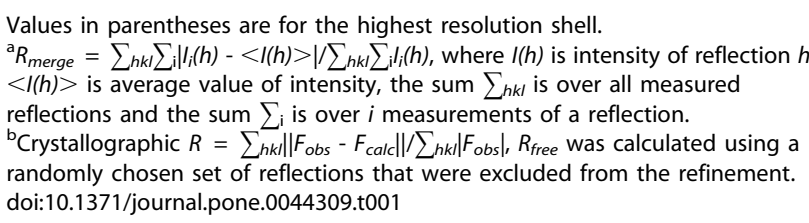 } \\
\hline
\end{tabular}

macroscopically as the result of the extraction of a thin "wedge" between subunits on the outer side of the ring, has the effect of decreasing the angle that a single subunit contributes towards the full $360^{\circ}$.

Taking our investigation a step further, we focused further studies at determining whether there is a general relationship between the oligomeric state of TRAP and its thermal stability, hence exploring factors that are not species-specific. The scenario found in the 12-mer $B$. halodurans TRAP, regarding the exclusion of the C-terminus, could logically be mimicked by truncating the 4 or $5 \mathrm{C}$-terminal residues, removing this segment from the subunit interface. This is observed in the previously determined structures of B. stearothermophilus E71stop TRAP and B. subtilis K71stop TRAP [9]. With respect to thermal stability accompanying the 11mer to 12-mer transition, we find that this truncation results in an increase in melting temperature, so that at $50 \mu \mathrm{M}$ L-tryptophan the increase in melting temperature between $B$. stearothermophilus wild type and E7lstop is $6^{\circ} \mathrm{C}$, from $81.4^{\circ} \mathrm{C}$ to $87.4^{\circ} \mathrm{C}$. The equivalent increase between $B$. subtilis wild type and $\mathrm{K} 71$ stop is $18.7^{\circ} \mathrm{C}, 44.3^{\circ} \mathrm{C}$ to $63.0^{\circ} \mathrm{C}$. This hints at an increase in thermal stability in response to incorporation of an additional subunit into the ring. However, this difference in melting temperature could also be a result of the absence of the $\mathrm{C}$-terminal residues themselves. For instance, we note that both $B$. subtilis and $B$. stearothermophilus wild type TRAP contain two flexible lysine residues at their G-termini [17] that can contribute electrostatically to the overall stability of TRAP. To understand which of these two factors - C-terminus or oligomeric state - has the greater influence we compared thermal stability of wild type $B$. subtilis TRAP 11mer with that of the B. subtilis S72N TRAP 12-mer, which still possesses the C-terminal segment but has it excluded from the subunit interface for steric reasons.

\section{S72N Mutation also Increases Subunit Number and Stability}

Exclusion of the G-terminus from the subunit interface can be achieved by introducing steric strain at a key region towards the $\mathrm{C}$ terminus that causes the segment to pivot out. In this respect, exclusion of the C-terminus in $\mathrm{S} 72 \mathrm{~N}$ is achieved in a manner more similar to how exclusion is achieved in B. halodurans TRAP. Residue 72 in particular (Ser 72 in B. subtilis TRAP) - which sits at the subunit-subunit interface in both wild type 11-mer structures studied - serves as a critical determinant in the conformation of the C-terminus. This is evident from the crystal structure presented in this study. From the structure of the S72N TRAP we see that substituting serine 72 for a bulkier amino acid, asparagine, does indeed introduce the necessary constraints to exclude the subsequent segment from the subunit interface. The thermal stability of this mutant 12-mer TRAP, S72N, is higher than that of its wild type 11-mer counterpart. For example at $50 \mu \mathrm{M}$ L-tryptophan the melting temperature of the wild type and mutant oligomers are $44 .{ }^{\circ} \mathrm{C}$ and $54.5^{\circ} \mathrm{C}$ respectively, yielding a stabilization of $10.2^{\circ} \mathrm{C}$. This is significant, albeit it is less compared to the $18.7^{\circ} \mathrm{C}$ stabilization we see from C-terminal truncation (i.e. B. subtilis $\mathrm{K} 71$ stop).

We conclude that by inducing an expansion in the oligomeric state from 11 to 12 subunits the thermal stability of the oligomer is increased. This increase is predominantly a result of the oligomeric state transition, and to a much lesser extent the influence of Cterminal or other specific residues. A potential, general relationship in TRAP oligomers therefore exists, in which melting temperature depends on subunit number. Further studies should be focused on evaluation of influence of the oligomeric state of TRAP on its activity in vivo.

\section{Binding Affinity Towards L-tryptophan}

To explore whether the stabilization by L-tryptophan was directly related to the affinity of TRAP oligomers to the binding of L-tryptophan, dissociation constants were measured. Results showed no obvious correlation with thermal stability or subunit number (Table 2). Although between B. subtilis wild type and S72N mutant TRAP, the 11-mer to 12-mer transition does coincide with a slight increase in affinity for L-tryptophan, these changes are only twofold, implying there are other more significant influences. 

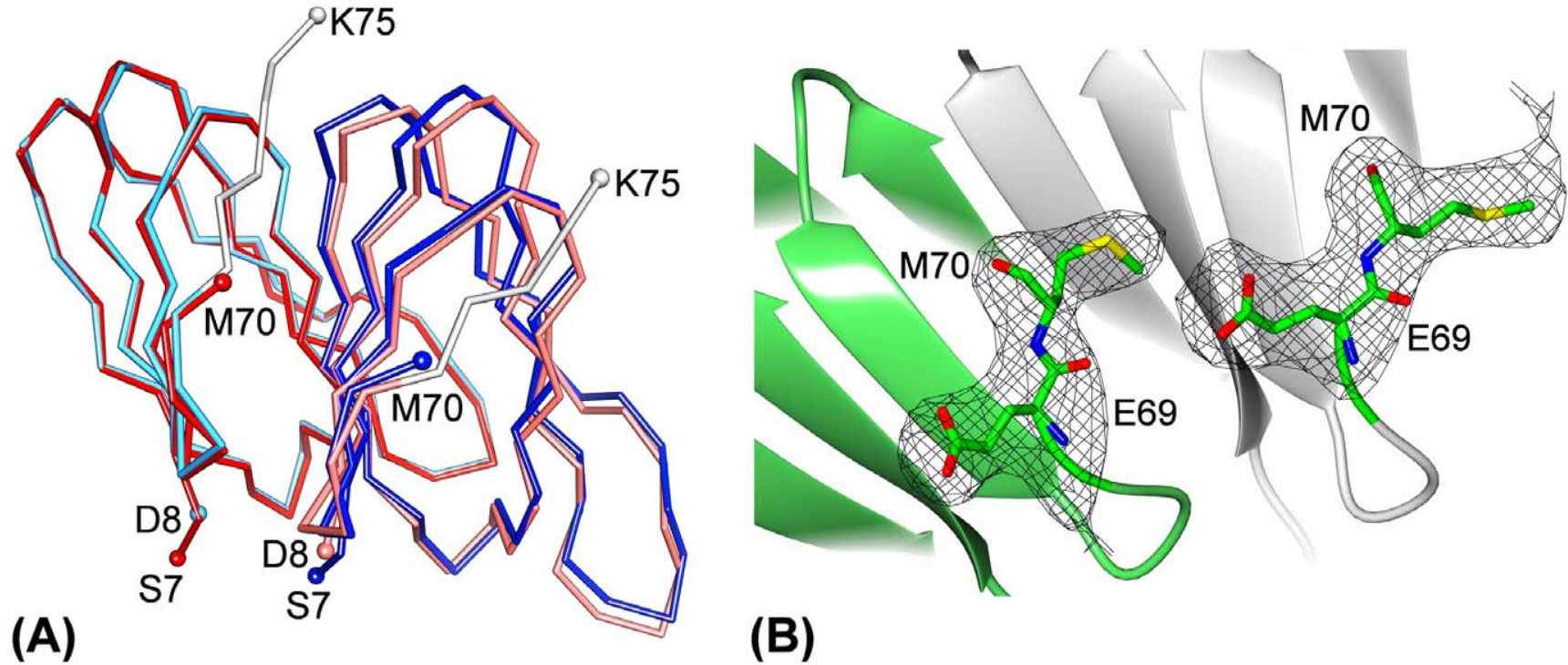

Figure 2. Structural differences between 11-subunit and 12-subunit TRAP. (A) Comparison of the B. subtilis wild type (red) and S72N mutant TRAP (blue). Two neighboring subunits were least-square fitted using main chain atoms of the subunit on the left. The C-terminal residues that are pivoted out of the subunit interface in S72N TRAP are highlighted in white on the wild type TRAP, starting from residue 69. (B) C-terminal residues E69 and $\mathrm{M70}$ are shown in sticks with main chain in yellow and side chains in turquoise, the rest of each subunit is shown in ribbons. The weighted $F_{\mathrm{o}}-F_{\mathrm{c}}$ omit maps, contoured at $2 \sigma$, were calculated after omitting residues 69 and 70 from the final model and 10 cycles of refinement. doi:10.1371/journal.pone.0044309.g002

\section{Increasing L-tryptophan Concentration Increases Thermal Stability}

For most TRAP oligomers studied we observe an increase in melting temperature in response to an increase in L-tryptophan concentration from $0 \mu \mathrm{M}$ to $100 \mu \mathrm{M}$ added. The trend does not appear in B. subtilis wild type TRAP however, over the Ltryptophan concentration range studied. Most concentrations in the monitored range represent many times the typical $\mathrm{K}_{\mathrm{d}}$ value,

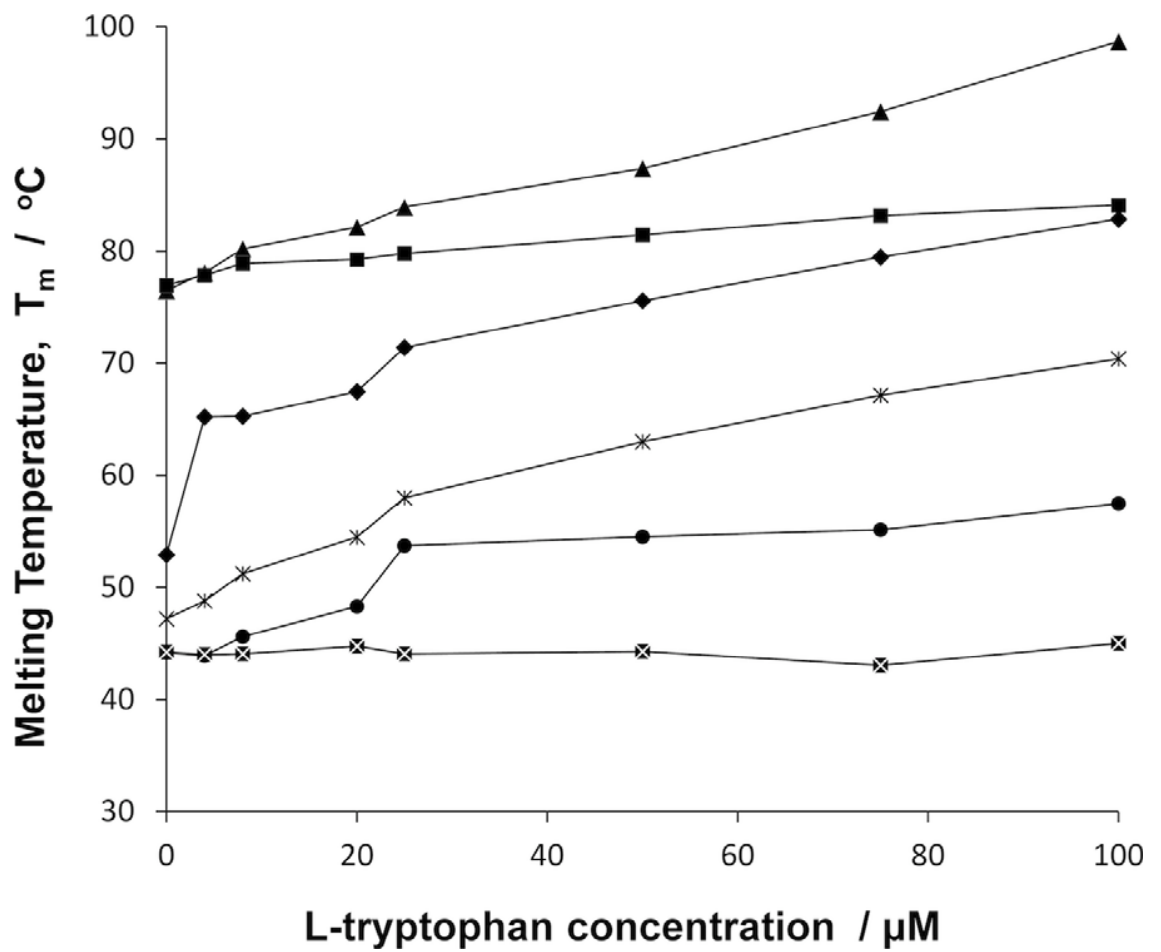

Figure 3. Melting temperatures of different TRAP oligomers. The dependence of melting temperature is shown for six TRAP oligomers, as a function of L-tryptophan concentration, determined by dye-based scanning fluorimetry. From the top: B. stearothermophilus E71stop 12-mer (triangle), B. stearothermophilus wild type 11-mer (square), B. halodurans wild type 12-mer (diamond), B. subtilis K71stop 12-mer (open cross), B. subtilis S72N 12-mer (circle), B. subtilis wild type 11-mer (crossed square). doi:10.1371/journal.pone.0044309.g003 
Table 2. L-tryptophan binding constants $\left(K_{d}\right)$ of three wild type and three mutant TRAP.

\begin{tabular}{lll}
\hline TRAP oligomer & Number of subunits & $\mathbf{K}_{\mathbf{d}} / \mu \mathbf{M}$ \\
\hline B. halodurans wild type & 12 & 4.5 \\
B. stearothermophilus wild type & 11 & 3.2 \\
B. stearothermophilus E71stop & 12 & 3.9 \\
B. subtilis wild type & 11 & 6.9 \\
B. subtilis K71stop & 12 & 6.5 \\
B. subtilis S72N & 12 & 2.8 \\
\hline doi:10.1371/journal.pone.0044309.t002 &
\end{tabular}

hence L-tryptophan binding-sites in TRAP are expected to be saturated. This therefore suggests a degree of non-specific binding of L-tryptophan to TRAP as the principal cause of the stabilization, over a thermodynamic or kinetic influence involving the L-tryptophan binding sites. It is likely that non-specific binding, beyond the 1:1 stoichiometry, would hence increase with increasing concentration of L-tryptophan.

\section{Conclusions}

This study demonstrates how the oligomeric state of a circular protein can be changed by introducing a point mutation at a subunit interface. Importantly, we demonstrate that a change in subunit number changes the stability of a circular assembly in vitro. In the case of TRAP, used in our studies, an increase in subunit number increases the stability for the oligomeric assembly. Predictably, this stability is dependent on solvent conditions, specifically L-tryptophan concentration. We conclude that a similarly subtle approach could be used during protein engineering of other ring-like structures, for stabilizing a particular oligomeric state thereby modifying dimensions, symmetry and stability.

\section{Materials and Methods}

\section{Gene Cloning, Protein Purification, Crystallization and Data Collection}

The B. subtilis $\mathrm{S} 72 \mathrm{~N}$ mutant TRAP was generated using the QuikChange kit (Stratagene, US) and a pET9a plasmid containing the wild type gene. S72N TRAP proteins were produced and purified as described previously [18]. Before crystallization, protein samples were transferred into solution containing $2 \mathrm{mM}$ Tris (pH 8.5), $300 \mathrm{mM} \mathrm{NaCl}$ and purified by size-exclusion chromatography using Superdex 200 Column (GE healthcare, UK). TRAP samples were initially analysed by size-exclusion chromatography, employing a Superdex 200 10/300 column (GE healthcare, UK) for the preliminary oligomeric assembly analysis. A 200-300 $\mu \mathrm{g}$ TRAP sample was injected and eluted by a buffer containing $20 \mathrm{mM}$ Tris-HCl, $30 \mathrm{mM} \mathrm{NaCl}$, and $0.1 \mathrm{mM} \mathrm{L}-$ tryptophan (pH 8.5). TRAP samples with an elution volume less than $14.5 \mathrm{ml}$ were selected for further analysis by native mass spectrometry analysis (Figure S1) and X-ray crystallographic studies.

Crystallization was carried out at $18^{\circ} \mathrm{C}$ by hanging drop vapor diffusion. For crystallization, $B$. subtilis S72N TRAP was transferred into solution containing $10 \mathrm{mM}$ triethanolamine (pH 8.0), $100 \mathrm{mM} \mathrm{NaCl}, 15 \mathrm{mM}$ L-tryptophan and concentrated to $33.5 \mathrm{mg} / \mathrm{ml}$. The reservoir contained $100 \mathrm{mM} \mathrm{Bis-Tris}$ propane (pH 8.5), $200 \mathrm{mM} \mathrm{KSCN}$ and 13\% PEG 3350 (v/v). Protein crystals were frozen using solutions containing all the crystallization ingredients with addition of $20 \%$ glycerol (v/v). The X-ray data were collected at $120 \mathrm{~K}$ at I04 beamline at the Diamond Light Source. Data were processed using HKL2000 [19], (Table 1).

\section{Structure Determination and Refinement}

All crystallographic calculations were carried out using the CCP4 program package [20]. The structure was determined by molecular replacement using MOLREP [21] with three adjacent subunits of $B$. stearothermophilus TRAP as a search model. Refinement was performed by REFMAC [22] and model rebuilding was done using COOT [23]. Water molecules were added automatically with the program ARP/wARP [24] and further corrected using maximum likelihood-weighted $2\left|\mathrm{~F}_{\mathrm{o}}\right|$ $\left|F_{c}\right|$ and $\left|F_{o}\right|-\left|F_{c}\right|$ electron density maps. Molecular contacts between adjacent monomers of TRAP were examined by CONTACT [20]. Figure 1 was generated using Bobscript [25], Figure 2A using UCSF Chimera [26], and Figure 2B using CCP4 mg [27].

\section{Dye-based Scanning Fluorimetry}

Dye-based scanning fluorimetry, or the Thermofluor method, was performed as previously described [28,29]. Protein samples were prepared by transferring to $50 \mathrm{mM}$ Tris $\mathrm{pH} 8,150 \mathrm{mM}$ $\mathrm{NaCl}, 10 \mathrm{mM} \mathrm{MgCl}_{2}$, with variable L-tryptophan concentration, in $30 \mathrm{kDa}$ cut-off Vivaspin concentrators, and diluting to $1 \mathrm{mg} /$ $\mathrm{ml}$, monitoring concentration by the Comassie-blue Bradford method. L-tryptophan concentration was varied from 0 to $0.1 \mathrm{mM}$ (final well concentration). Samples were transferred to 96-well thin-walled clear PCR plates. Each well contained $30 \mu \mathrm{l}$ of solution, consisting of $15 \mu \mathrm{l}$ of the $1 \mathrm{mg} / \mathrm{ml}$ protein (yielding a final concentration of $0.5 \mathrm{mg} / \mathrm{ml}), 1 / 1000$ diluted SYPRO Orange (Sigma-Aldrich) and $50 \mathrm{mM}$ Tris $\mathrm{pH} 8$ with a specified L-tryptophan concentration. A Stratagene Mx3005P QPGR instrument was programmed to increase temperature in $1{ }^{\circ} \mathrm{C}$ increments at 30 second intervals, from $25^{\circ} \mathrm{C}$ to $95^{\circ} \mathrm{C}$ with $470 \mathrm{~nm}$ excitation, measuring fluorescence at $570 \mathrm{~nm}$.

\section{Measurement of L-tryptophan Dissociation Constants}

L-tryptophan binding to TRAP was measured using a fluorescence assay based on competition between L-tryptophan and 1-anilionaphthalene-8-sulfonic acid (ANS) as described previously [30]. ANS fluoresces weakly in solution but fluoresces strongly at $460 \mathrm{~nm}$ with $372 \mathrm{~nm}$ excitation upon binding to TRAP. L-tryptophan has a greater affinity than ANS for TRAP, and displaces ANS thus reducing the fluorescence. The change in fluorescence, $\Delta \mathrm{F}$, was monitored using a LS-50B fluorometer (Perkin Elmer). A $100 \mu \mathrm{L}$ sample of $15 \mu \mathrm{M}$ TRAP was prepared in $60 \mu \mathrm{M}$ ANS and $0.5 \mathrm{mM}$ sodium phosphate at $\mathrm{pH} 8.0$. Readings of fluorescence intensity were taken from 0 to $2000 \mu \mathrm{M}$ tryptophan. At each concentration of tryptophan, fluorescence intensity was collected at room temperature for one minute to allow the reaction to reach equilibrium. Fluorescence intensity was averaged for each concentration of L-tryptophan and then used to calculate the change in fluorescence $(\Delta \mathrm{F})$. The maximal change in fluorescence was set to $100 \%$ and values of $\Delta \mathrm{F}$ were normalised accordingly. GraphPad Prizm 4 was used to plot $\% \Delta \mathrm{F}$ as a function of tryptophan concentration to obtain L-tryptophan dissociation constants and binding curves. 


\section{Data Deposition}

Refined coordinates of the B. subtilis S72N TRAP and structure factors have been deposited with the Protein Data Bank under accession code $4 \mathrm{~B} 27$.

\section{Supporting Information}

Figure S1 Native mass (Nanoflow electrospray) spectrum of $B$. subtilis $\mathbf{S} 72$ NRAP. $\mathrm{m} / \mathrm{z}$ corresponds to the massto-charge ratio. Red hexagons correspond to 12-mer TRAP species without bound tryptophan.

(DOCX)

Figure S2 Melting curves of $B$. halodurans and $B$. stearothermophilus wild type TRAP. Normalised fluorescence data $(\mathbf{O})$ are overlaid with CD data recorded at $205 \mathrm{~nm}(\boldsymbol{\square})$, both at $50 \mu \mathrm{M}$ L-tryptophan in the sample. (A) B. halodurans wild type TRAP (12-mer). (B) B. stearothermophilus wild type TRAP (11mer). Sigmoidal curves were fitted to CD spectroscopy data (red) for comparison against fluorescence data. The melting temperatures represented by the midpoints of these curves were found to be $78.5^{\circ} \mathrm{C}$ and $81.6^{\circ} \mathrm{C}$ for $(\mathrm{A})$ and $(\mathrm{B})$ respectively. These compare well with the melting temperatures derived from curves fitted to the fluorescence data, $75.6^{\circ} \mathrm{C}$ and $81.4^{\circ} \mathrm{C}$ respectively (Table $\mathrm{S} 1$ ). (DOCX)

Figure S3 (A) Overlays of 8 normalised fluorescence versus temperature curves of $B$. stearothermophilus E71Stop TRAP, with concentration of L-tryptophan increasing left to right with lightening shades of green: $0,4,8,20,25,50,75,100 \mu \mathrm{M}$. (B) Curves derived from four replicates at two concentrations of Ltryptophan, for $B$. stearothermophilus E71Stop TRAP: $0 \mu \mathrm{M} \mathrm{L-}$

\section{References}

1. Minamino T, Imada K, Kinoshita M, Nakamura S, Morimoto YV, et al. (2011) Structural Insight into the Rotational Switching Mechanism of the Bacterial Flagellar Motor. PLoS Biology 9(5): e1000616.

2. Weber J, Senior AE (2003) ATP synthesis driven by proton transport in F1F0ATP synthase. Febs Letters 545(1): 61-70.

3. Rao VB, Feiss M (2008) The Bacteriophage DNA Packaging Motor. Annual Review of Genetics 42: 647-81.

4. Casjens SR (2011) The DNA-packaging nanomotor of tailed bacteriophages. Nature Reviews Microbiology 9(9): 647-57.

5. Lebedev AA, Krause MH, Isidro AL, Vagin AA, Orlova EV, et al. (2007) Structural framework for DNA translocation via the viral portal protein. Embo Journal 26(7): 1984-94.

6. Thomas DR, Francis NR, Xu C, DeRosier DJ (2006) The three-dimensional structure of the flagellar rotor from a clockwise-locked mutant of Salmonella enterica serovar typhimurium. Journal of Bacteriology 188(20): 7039-48.

7. Wendell D, Jing P, Geng J, Subramaniam V, Lee TJ, et al. (2009) Translocation of double-stranded DNA through membrane-adapted phi29 motor protein nanopores. Nature Nanotechnology 4(11): 765-72.

8. Antson AA, Dodson EJ, Dodson G, Greaves RB, Chen XP, et al. (1999) Structure of the $\operatorname{tr} p$ RNA-binding attenuation protein, TRAP, bound to RNA. Nature 401(6750): 235-42.

9. Chen C-S, Smits C, Dodson GG, Shevtsov MB, Merlino N, et al. (2011) How to Change the Oligomeric State of a Circular Protein Assembly: Switch from 11Subunit to 12-Subunit TRAP Suggests a General Mechanism. PLoS ONE 6(10): e25296.

10. Gollnick P, Babitzke P, Antson A, Yanofsky C (2005) Complexity in regulation of tryptophan biosynthesis in Bacillus subtilis. Annual Review of Genetics 39: 47-68.

11. Heddle JG, Fujiwara I, Yamadaki H, Yoshii S, Nishio K, et al. (2007) Using the ring-shaped protein TRAP to capture and confine gold nanodots on a surface. Small 3(11): 1950-6.

12. Miranda FF, Iwasaki K, Akashi S, Sumitomo K, Kobayashi M, et al. (2009) A Self-Assembled Protein Nanotube with High Aspect Ratio. Small 5(18): 207784.

13. McElroy CA, Manfredo A, Gollnick P, Foster MP (2006) Thermodynamics of tryptophan-mediated activation of the $\operatorname{trp}$ RNA-binding attenuation protein. Biochemistry 45(25): 7844-53.

14. McElroy C, Manfredo A, Wendt A, Gollnick P, Foster M (2002) TROSY-NMR studies of the $91 \mathrm{kDa}$ TRAP protein reveal allosteric control of a gene regulatory tryptophan $($ dark red $=$ mean; pink $=$ replicates $), 50 \mu \mathrm{M}$ L-tryptophan $($ dark blue $=$ mean; light blue $=$ replicates $)$, normalised to the mean curve, showing equivalent midpoint and plateau positions within replicates.

(DOCX)

Table S1 Melting temperatures for TRAP oligomers over 0 to 100 mM L-tryptophan concentration. $B$. halo. (B. halodurans); B. stearo. (B.stearothermophilus); B. sub. (B. subtilis); WT (wild type). Standard deviations across four repeated measurements are shown in the parentheses.

(DOCX)

Text S1 Materials and Methods - Native Mass Spectrometry.

(DOCX)

Text S2 Materials and Methods - Gircular Dichroism spectroscopy.

(DOCX)

\section{Acknowledgments}

The authors thank Sam Hart and Johan Turkenburg for help during the X-ray data collection and acknowledge support of the Diamond Light Source.

\section{Author Contributions}

Conceived and designed the experiments: OWB C-SC WL CS PG AAA. Performed the experiments: OWB C-SC ARP WL. Analyzed the data: OWB C-SC ARP CS PG AAA. Wrote the paper: OWB C-SC ARP CS PG AAA.

protein by ligand-altered flexibility. Journal of Molecular Biology 323(3): 46373.

15. Malay AD, Watanabe M, Heddle JG, Tame JRH (2011) Grystal structure of unliganded TRAP: implications for dynamic allostery. Biochemical Journal 434: 427-34.

16. Ruotolo BT, Giles K, Campuzano I, Sandercock AM, Bateman RH, et al. (2005) Evidence for macromolecular protein rings in the absence of bulk water. Science 310(5754): 1658-61.

17. Chen XP, Antson AA, Yang M, Li P, Baumann C, et al. (1999) Regulatory features of the trp operon and the crystal structure of the trp RNA-binding attenuation protein from Bacillus stearothermophilus. Journal of Molecular Biology 289(4): 1003-16.

18. Antson AA, Otridge J, Brzozowski AM, Dodson EJ, Dodson GG, et al. (1995) The structure of trp RNA-binding attenuation protein. Nature 374(6524): 693700 .

19. Otwinowski Z, Minor W (1997) Processing of X-ray diffraction data collected in oscillation mode. Macromolecular Crystallography, Pt A 276: 307-26.

20. Bailey S (1994) The CCP4 Suite - programs for protein crystallography. Acta Crystallographica Section D-Biological Crystallography. 50: 760-3.

21. Vagin A, Teplyakov A (2000) An approach to multi-copy search in molecular replacement. Acta Crystallographica Section D-Biological Crystallography 56: $1622-4$.

22. Murshudov GN, Vagin AA, Lebedev A, Wilson KS, Dodson EJ (1999) Efficient anisotropic refinement of macromolecular structures using FFT. Acta Crystallographica Section D - Biological Crystallography 55: 247-55.

23. Emsley P, Cowtan K (2004) Coot: model-building tools for molecular graphics. Acta Crystallographica Section D - Biological Crystallography 60: 2126-32.

24. Lamzin VS, Wilson KS (1993) Automated refinement of protein models. Acta Crystallographica Section D - Biological Crystallography 49: 129-47.

25. Esnouf RM (1997) An extensively modified version of MolScript that includes greatly enhanced coloring capabilities. Journal of Molecular Graphics \& Modelling 15(2): 132-134.

26. Pettersen EF, Goddard TD, Huang GC, Couch GS, Greenblatt DM, et al. (2004) UCSF Chimera - A visualization system for exploratory research and analysis. Journal of Computational Chemistry 25(13): 1605-12.

27. Potterton L, McNicholas S, Krissinel E, Gruber J, Cowtan K, et al. (2004) Developments in the CCP4 molecular-graphics project. Acta Crystallographica Section D - Biological Crystallography 60: 2288-94. 
28. Niesen FH, Berglund H, Vedadi M (2007) The use of differential scanning fluorimetry to detect ligand interactions that promote protein stability. Nature Protocols 2(9): 2212-21.

29. Ericsson UB, Hallberg BM, DeTitta GT, Dekker N, Nordlund P (2006) Thermofluor-based high-throughput stability optimization of proteins for structural studies. Analytical Biochemistry 357(2): 289-98.
30. Li PTX, Gollnick P (2004) Characterization of a trp RNA-binding attenuation protein (TRAP) mutant with tryptophan independent RNA binding activity. Journal of Molecular Biology 335(3): 707-22. 